\title{
Dietary Treatment in Children with Eosinophilic Oesophagitis
}

\section{Chandra Sekhar Devulapalli}

Senior Medical Consultant and Paediatrician, Norwegian Labour and Welfare Administration (NAV), work and benefits Kristiania, Oslo, Norway

\section{Introduction}

Eosinophilic oesophagitis (EoE) is diagnosed in an increasing number of children, but it is uncertain whether this is due to increased prevalence or increased awareness among physicians. EoE is characterized by chronic inflammation and infiltration of eosinophilic granulocytes into the oesophagus and is related to food allergies. The youngest children most often have symptoms that may be reminiscent of gastroesophageal reflux disease - eating disorder and poor growth in infancy and eventually gulping, vomiting and abdominal pain. During childhood, vomiting and / or abdominal pain or retrosternal pain are reported, while during adolescence, symptoms of reflux disease, dysphagia and fasting foods are the most frequent symptoms [1,2]. In many, the mechanism is probably an allergic reaction in the oesophagus to some foods, and over half of patients have an atopic disposition. The diagnosis is based on clinical symptoms, presence of eosinophilic granulocytes in the oesophagus and typical endoscopic findings.

Treatment options for EoE in children mainly include elimination diets and locally acting corticosteroids and endoscopic dilatation [13]. To avoid unnecessary elimination diets and to identify children with proton pump inhibitor (PPI) -responsive EoE, an 8 week PPI trial $[4,5]$ is recommended. This should then be followed by endoscopic and histological reassessment, regardless of whether there is symptom relief or not. If eosinophilic inflammation persists after PPI treatment and other causes of EoE are less likely, the diagnosis of EoE can be confirmed [4]. In patients with EoE, where PPI has no effect, an elimination diet and / or with locally acting steroids may be attempted $[4,5]$.

\section{Elimination Diet}

Importance of foods in the disease was understood after studies in children with EoE who got rid of their ailments when treated with the elemental diet, a purely amino acid-based regimen without allergenic properties [6-8]. The diet led to histological normalization of the oesophagus in most of the children. Reintroduction of specific foods resulted in recurrence of the ailments. Results of the allergy tests are used as a starting point to discuss any elimination diet with motivated patients and families. This is mainly of two ways: targeted elimination diet which removes only suspected allergens or empirical elimination diet where one removes the most common allergens.

Elimination diet can be challenging for patient and parents, and requires careful follow-up by the nutritionist. Targeted elimination diet involves removing foods that are suspected either on the basis of history or allergy tests. In addition, it is recommended that one removes milk from the diet anyway, as this is the most common allergen. After 8-12 weeks, new oesophagoscopy is performed with biopsies, and by histological remission, one by one food is reintroduced. Some will have the effect of removing a particular food, while some will need to remove many $[4,5]$. Empirical elimination diet ("6 food elimination diet") is a practical form of elimination diet where common allergenic foods such as milk, eggs, wheat, peanuts, soy and seafood are excluded from the diet for 8-12 weeks. This should be followed by endoscopy

\section{Publication History:}

Received: April 01, 2020

Accepted: April 24, 2020

Published: April 26, 2020

\section{Keywords:}

Eosinophilic Oesophagitis, Elimination, Endoscopic dilatation

with biopsies. This approach might lead to symptomatic improvement in about $70 \%$. This has proven to be as effective as an elimination diet based on skin tests and measurement of specific IgE [2,3]. After histological remission, a reintroduction of food starts, one by one until one finds the food (s) that trigger the disease [4,5].

There is an increasing proportion of applications for basic benefit due to EoE in children when the diet should be free of wheat for a certain period or on a permanent basis. Patients with this condition are often in the testing phase where several other foods are eliminated, as part of the elimination diet [4-6]. Elimination of certain foods can lead to histological and symptomatic improvement. However, identifying which foods should be eliminated in the diet can be challenging. Although the condition is chronic, many have long and good periods. It is currently unclear how long patients should have such a strict elimination diet. The severity of the symptoms rarely justifies a very restrictive diet [3]. Dietary treatment in children must be done in close collaboration with the clinical nutritionist since restrictive diets can be difficult to follow and lead to reduced quality of life and malnutrition. Treatment must be tailored and individualized.

\section{Competing Interests}

The author declare no competing interests.

\section{References}

1. Spergel JM, Brown-Whitehorn TF, Beausoleil JL, Franciosi J, Shuker M, et al. (2009) 14 years of eosinophilic esophagitis: clinical features and prognosis. J Pediatr Gastroenterol Nutr 48: 30-36.

2. Liacouras CA, Furuta GT, Hirano I, Atkins D, Attwood SE, et al. (2011) Eosinophilic esophagitis: updated consensus recommendations for children and adults. J Allergy Clin Immunol 128: 3-20.e6.

3. Holme $\varnothing$, Tjora E, Aabakken L (2011) [Eosinophil oesophagitis]. Norwegian. Tidsskr Nor Laegeforen 131: 2470-2474.

4. Papadopoulou A, Koletzko S, Heuschkel R, Dias JA, Allen KJ, et al. (2014) Management guidelines of eosinophilic esophagitis in childhood. J Pediatr Gastroenterol Nutr 58:107-118.

*Corresponding Author: Dr. Chandra Sekhar Devulapalli, Senior Medical Consultant and Paediatrician, Norwegian Labour and Welfare Administration (NAV), work and benefits Kristiania, Oslo, Norway; E-mail: chandev@gmail.com

Citation: Devulapalli CS (2020) Dietary Treatment in Children with Eosinophilic Oesophagitis. Int J Pediatr Neonat Care 6: 164. doi: https://doi.org/10.15344/24552364/2020/164

Copyright: (C) 2020 Devulapalli. This is an open-access article distributed under the terms of the Creative Commons Attribution License, which permits unrestricted use, distribution, and reproduction in any medium, provided the original author and source are credited. 
Citation: Devulapalli CS (2020) Dietary Treatment in Children with Eosinophilic Oesophagitis. Int J Pediatr Neonat Care 6: 164. doi: https://doi.org/10.15344/24552364/2020/164

Page 2 of 2

5. Lucendo AJ, Molina-Infante J, Arias Á, von Arnim U, Bredenoord AJ, et al (2017) Guidelines on eosinophilic esophagitis: evidence-based statements and recommendations for diagnosis and management in children and adults. United European Gastroenterol J 5: 335-358

6. Kelly KJ, Lazenby AJ, Rowe PC, Yardley JH, Perman JA, et al. (1995) Eosinophilic esophagitis attributed to gastroesophageal reflux: improvement with an amino acid-based formula. Gastroenterology 109: 1503-1512.

7. Markowitz JE1, Spergel JM, Ruchelli E, Liacouras CA (2003) Elemental diet is an effective treatment for eosinophilic esophagitis in children and adolescents. Am J Gastroenterol 98: 777-782.

8. Spergel JM, Andrews T, Brown-Whitehorn TF, Beausoleil JL, Liacouras CA (2005) Treatment of eosinophilic esophagitis with specific food elimination diet directed by a combination of skin prick and patch tests. Ann Allergy Asthma Immunol 95: 336-43. 